\title{
BMC Cardiovascular Disorders reviewer acknowledgement 2014
}

Tim Shipley

\section{Contributing reviewers}

The editors of BMC Cardiovascular Disorders would like to thank all our reviewers who have contributed to the journal in Volume 14 (2014).

Pedro Abizanda
Spain

Vikram Agarwal

United States of America

Umme Aiman

India

Muharrem Akin

Germany

Majd Al Ghatrif

United States of America

Stephane Allouche

France

Abdallah Al-Mohammad

United Kingdom

Alvaro Alonso

United States of America

Bahaaldin Alsoufi

United States of America

Rachel Amiya

Japan

Enrico Ammirati

Italy

Daraban Ana Maria

Romania
Ajith Ananthakrishna Pillai
India

Annalisa Angelini

Italy

Emiliano Angeloni

Italy

Uzair Ansari

Germany

Pamella Anunciação

Brazil

Ashraf Anwar

Saudi Arabia

Yaron Arbel

Israel

Carmelina Ariano

Italy

Rakesh Arora

Canada

Dharamvir Arya

India

Kei Asayama

Belgium

Ajax Atta

Brazil
Wael Attia

Egypt

Johann Auer

Austria

Anna Axelsson

Denmark

Flavia Ballocca

Italy

Sevket Balta

Turkey

Frédéric Banal

France

Marco Barbanti

Italy

Umberto Barbero

Italy

Maria Barcelo

Spain

Fellype Barreto

Brazil

Pier Paolo Bassareo

Italy

Rob Bauer

United States of America

Correspondence: tim.shipley@biomedcentral.com

BioMed Central, Floor 6, 236 Gray's Inn Road, London WC1X 8HB, United

Kingdom

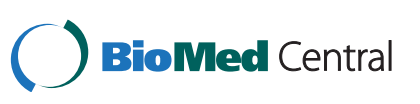


Chirag Bavishi

United States of America

\section{Alexander Becker \\ Germany \\ Hassan Behlouli \\ Canada}

Michael Behnes

Germany

Iddo Ben-Dov

Israel

Kálmán Benke

Hungary

Knut Erik Berge

Norway

Alessandro Bernardi

Italy

Ishir Bhan

United States of America

Kartikeya Bhargava

India

Jacek Bialkowski

Poland

\section{Matteo Bianco}

Italy

Mandy Binning

United States of America

Federico Bizzarri

Italy

Emanuela Bostjancic

Slovenia

Marouane Boukhris

Tunisia

Marc Buijsrogge

Netherlands

Alida Caforio

Italy

Adriano Caixeta

Brazil

Gianluca Campo

Italy

Ahmet Selçuk Can

Turkey

$\mathrm{Xu}$ Cao

United States of America
Marina Cerbo

Italy

John Chambers

United Kingdom

Steven Chamuleau

Netherlands

Hoi Chan

United States of America

Po-Yuan Chang

Taiwan

Meng-Hua Chen

China

Shaojie Chen

China

Hsin-Jen Chen

United States of America

Xiangmei Chen

China

Benjamin Cheong

United States of America

Jae Hyung Cho

United States of America

Rocky Chow

Taiwan

Imke Christiaans

Netherlands

Thomas Christophersen

Denmark

Pao-Hsien Chu

Taiwan

Siew Siang Chua

Malaysia

Shao-Yuan Chuang

Taiwan

Antonio Ciccarelli

Italy

Alexander Clark

Canada

Aldo Clerico

Italy

Sean Coady

United States of America

Steven Coca

United States of America
Dennis Cokkinos

Greece

Federico Conrotto

Italy

Adolfo Correa

United States of America

Xenophon Costeas

Greece

Antonio Crisafulli

Italy

Agnieszka Cudnoch-Jedrzejewska

Poland

Douglas Curran-Everett

United States of America

Cesare Cuspidi

Italy

Saleem Dabbah

Israel

Hiroyuki Daida

Japan

Fabrizio D'Ascenzo

Italy

Linda De Heer

Netherlands

Wouter De Ruijter

Netherlands

Renato De Vecchis

Italy

Kevin Deans

United Kingdom

Michael Debney

United Kingdom

Jean-Marie Degryse

Belgium

Victoria Delgado

Netherlands

Christian Delles

United Kingdom

Yvan Devaux

Luxembourg

Permphan Dharmasaroja

Thailand

Giuseppina Di Biase

Italy 


\begin{tabular}{|c|c|c|}
\hline $\begin{array}{l}\text { Giovanni Di Salvo } \\
\text { Italy }\end{array}$ & $\begin{array}{l}\text { Sebastien Foulquier } \\
\text { Netherlands }\end{array}$ & $\begin{array}{l}\text { Wan-Jie Gu } \\
\text { China }\end{array}$ \\
\hline $\begin{array}{l}\text { Frank Dini } \\
\text { Italy }\end{array}$ & $\begin{array}{l}\text { Marcus Franz } \\
\text { Germany }\end{array}$ & $\begin{array}{l}\text { Andrey Gudkov } \\
\text { Russian Federation }\end{array}$ \\
\hline $\begin{array}{l}\text { James Di Nicolantonio } \\
\text { United States of America }\end{array}$ & $\begin{array}{l}\text { Marie Freel } \\
\text { United Kingdom }\end{array}$ & $\begin{array}{l}\text { Hartmut Karl Guelker } \\
\text { Germany }\end{array}$ \\
\hline $\begin{array}{l}\text { Torsten Doenst } \\
\text { Germany }\end{array}$ & $\begin{array}{l}\text { Lars Frost } \\
\text { Denmark }\end{array}$ & $\begin{array}{l}\text { Hervé Guillou } \\
\text { France }\end{array}$ \\
\hline $\begin{array}{l}\text { Rudin Domi } \\
\text { Albania }\end{array}$ & $\begin{array}{l}\text { Yoshihiro Fukumoto } \\
\text { Japan }\end{array}$ & $\begin{array}{l}\text { Oliver Gunkel } \\
\text { Germany }\end{array}$ \\
\hline $\begin{array}{l}\text { Chiara Donfrancesco } \\
\text { Italy }\end{array}$ & $\begin{array}{l}\text { Adelaide Fusco } \\
\text { Italy }\end{array}$ & $\begin{array}{l}\text { Mark Haigney } \\
\text { United States of America }\end{array}$ \\
\hline $\begin{array}{l}\text { Mei Dong } \\
\text { China }\end{array}$ & $\begin{array}{l}\text { Angelo Gaffo } \\
\text { United States of America }\end{array}$ & $\begin{array}{l}\text { Nobuyuki Hamajima } \\
\text { Japan }\end{array}$ \\
\hline $\begin{array}{l}\text { Rami Doukky } \\
\text { United States of America }\end{array}$ & $\begin{array}{l}\text { Ujjawal Gandhi } \\
\text { United States of America }\end{array}$ & $\begin{array}{l}\text { Job Harenberg } \\
\text { Germany }\end{array}$ \\
\hline $\begin{array}{l}\text { Danny Dvir } \\
\text { Canada }\end{array}$ & $\begin{array}{l}\text { Ron Gansevoort } \\
\text { Netherlands }\end{array}$ & $\begin{array}{l}\text { David Haslam } \\
\text { United Kingdom }\end{array}$ \\
\hline $\begin{array}{l}\text { Nicole Ebner } \\
\text { Germany }\end{array}$ & $\begin{array}{l}\text { Lei Gao } \\
\text { China }\end{array}$ & $\begin{array}{l}\text { Eleni Hatzinikolaou-Kotsakou } \\
\text { Greece }\end{array}$ \\
\hline $\begin{array}{l}\text { Elisa Ebrille } \\
\text { Italy }\end{array}$ & $\begin{array}{l}\text { Carlo Gaudio } \\
\text { Italy }\end{array}$ & $\begin{array}{l}\text { Jan Heeringa } \\
\text { Netherlands }\end{array}$ \\
\hline $\begin{array}{l}\text { Ulf Ekelund } \\
\text { Sweden }\end{array}$ & $\begin{array}{l}\text { J. Christoph Geller } \\
\text { Germany }\end{array}$ & $\begin{array}{l}\text { Kevin Heffernan } \\
\text { United States of America }\end{array}$ \\
\hline $\begin{array}{l}\text { John Elefteriades } \\
\text { United States of America }\end{array}$ & $\begin{array}{l}\text { Jeffrey Geske } \\
\text { United States of America }\end{array}$ & $\begin{array}{l}\text { Martin Holzmann } \\
\text { Sweden }\end{array}$ \\
\hline $\begin{array}{l}\text { Hazem Elewa } \\
\text { United States of America }\end{array}$ & $\begin{array}{l}\text { Sebastiano Gili } \\
\text { Italy }\end{array}$ & $\begin{array}{l}\text { Yuling Hong } \\
\text { United States of America }\end{array}$ \\
\hline $\begin{array}{l}\text { Salma Esmaeil } \\
\text { Egypt }\end{array}$ & $\begin{array}{l}\text { Thomas Gillette } \\
\text { United States of America }\end{array}$ & $\begin{array}{l}\text { Dongming Hou } \\
\text { United States of America }\end{array}$ \\
\hline $\begin{array}{l}\text { Rodrigo Estevez-Loureiro } \\
\text { Spain }\end{array}$ & $\begin{array}{l}\text { Francesca Giordana } \\
\text { Italy }\end{array}$ & $\begin{array}{l}\text { Sandy Hu } \\
\text { China }\end{array}$ \\
\hline $\begin{array}{l}\text { Leanne Felkin } \\
\text { United Kingdom }\end{array}$ & $\begin{array}{l}\text { Grégoire Girod } \\
\text { Switzerland }\end{array}$ & $\begin{array}{l}\text { Po Hsun Huang } \\
\text { Taiwan }\end{array}$ \\
\hline $\begin{array}{l}\text { Timothy Fendler } \\
\text { United States of America }\end{array}$ & $\begin{array}{l}\text { Stephen Glasser } \\
\text { United States of America }\end{array}$ & $\begin{array}{l}\text { Frank Hulstaert } \\
\text { Belgium }\end{array}$ \\
\hline $\begin{array}{l}\text { Ignacio Ferreira } \\
\text { Spain }\end{array}$ & $\begin{array}{l}\text { Tadhg Gleeson } \\
\text { Ireland }\end{array}$ & $\begin{array}{l}\text { Holger Husi } \\
\text { United Kingdom }\end{array}$ \\
\hline $\begin{array}{l}\text { Claudia Fiorina } \\
\text { Italy }\end{array}$ & $\begin{array}{l}\text { Sameer Goyal } \\
\text { India }\end{array}$ & $\begin{array}{l}\text { Olga Husson } \\
\text { Netherlands }\end{array}$ \\
\hline $\begin{array}{l}\text { Sara Fitzgerald-Butt } \\
\text { United States of America }\end{array}$ & $\begin{array}{l}\text { Anders M. Greve } \\
\text { Denmark }\end{array}$ & $\begin{array}{l}\text { Mario Iannaccone } \\
\text { Italy }\end{array}$ \\
\hline $\begin{array}{l}\text { Joost Fledderus } \\
\text { Netherlands }\end{array}$ & $\begin{array}{l}\text { Eric Gross } \\
\text { United States of America }\end{array}$ & $\begin{array}{l}\text { Motoyuki Iemitsu } \\
\text { Japan }\end{array}$ \\
\hline
\end{tabular}




\author{
Massimo Imazio \\ Italy \\ Hart Isaacs \\ United States of America \\ Yasushi Ishigaki \\ Japan \\ Yoshitaka Iwanaga \\ Japan
}

Raffaele Izzo

Italy

Frederic Jacques

Canada

Sae Young Jae

Korea, South

Piia Jallinoja

Finland

Ruben Jara-Rubio

Spain

Li Jia

China

Edward Johns

Ireland

Alfonso Jurado-Roman

Spain

Edo Kaluski

United States of America

Petra Kameritsch

Germany

Bharat Kantharia

United States of America

Mehran Karimi

Iran

Mahwash Kassi

United States of America

Hisashi Kawashima

Japan

Do Han Kim

Korea, South

Ajay Kirtane

United States of America

Soichiro Kitamura

Japan

Jesper Kjaergaard

Denmark
Julie Klein

France

Peter Klein-Weigel

Germany

Gert Klug

Austria

Tijmen Koëter

Netherlands

Andrew Kompa

Australia

Deling Kong

China

Alexandra Konradi

Russian Federation

David Koolbergen

Netherlands

Jelena Kornej

Germany

Johannes Kragten

Netherlands

Martin Krakauer

Denmark

Mahendra Kumar

United States of America

Dharam Kumbhani

United States of America

Sung Uk Kwon

Korea, South

Michael La Fountaine

United States of America

Chun-Fu Lai

Taiwan

Malinee Laopaiboon

Thailand

Camille Lassale

United Kingdom

Johan Lassus

Finland

Crystal Lee

Australia

Won Young Lee

Korea, South

Ju-Mi Lee

Korea, South
Jung Eun Lee

Korea, South

Yau-Jiunn Lee

Taiwan

Gilles Lemesle

France

Clive Lewis

United Kingdom

Hung-Yuan Li

Taiwan

Clare Liddy

Canada

Edgars Liepins

Latvia

Lian-Yu Lin

Taiwan

Lisa Lincz

Australia

Daniel Lingamfelter

United States of America

Eric Lopatta

Germany

Oscar Lorenzo

Spain

Eric Loucks

United States of America

Nicole Lowres

Australia

Xinwu Lu

China

Xiaofang Lu

United States of America

Andrew Ludman

United Kingdom

Joanne Luke

Australia

David Maciver

United Kingdom

Judith Mackall

United States of America

Jared Magnani

United States of America

Ahmed Mahmoud

United States of America 
Bernhard Maisch

Germany

Olebogeng Majane

South Africa

Bilal Majed

France

Shinji Makita

Japan

Boris A. Malyarchuk

Russian Federation

Nassos Manginas

Greece

Samer Mansour

Canada

Luis Manzano

Spain

Themistoklis Maounis

Greece

Germanas Marinskis

Lithuania

Patrick Mark

United Kingdom

Pedro Marques Da Silva

Portugal

Ruth Masterson Creber

United States of America

Guido A. Matschuck

Germany

Sophie Mavrogeni

Greece

Otto Mayer

Czech Republic

Agnes Mayr

Austria

Terence Mccann

Australia

Alex Mclellan

Australia

Nehal Mehta

United States of America

Puja Mehta

United States of America

Catharina Mels

South Africa
Jort Merken

Netherlands

Bernhard Metzler

Austria

Sven Meyer

Germany

Thomas Meyer

Germany

Antonio Miceli

Italy

Akiko Mii

Japan

Alma Mingels

Netherlands

Yoko Miyasaka

Japan

Pietro Amedeo Modesti

Italy

Misbahuddin Mohammad

United Kingdom

Francois-Pierre Mongeon

Canada

Nuno Moreno

Portugal

I Haldun Müderrisoglu

Turkey

Christian Mueller

Switzerland

Hans-Wilhelm Müller

Germany

Venkatesh Murthy

United States of America

Karthik Murugiah

United States of America

Hazumu Nagata

Japan

Maria Navas

United States of America

Isao Nishi

Japan

Marko Noc

Slovenia

Youssef Nosir

Saudi Arabia
Ntobeko Ntusi

United Kingdom

Evangelia Ntzani

Greece

Gaetano Nucifora

Italy

Naro Ohashi

Japan

Sadanori Okada

Japan

Takanobu Okamoto

Japan

Elena Okisheva

Russian Federation

Takafumi Okura

Japan

Altan Onat

Turkey

Michelle Orme

United Kingdom

Christian Ott

Germany

Linda Oude Griep

United Kingdom

Sara Palacio Restrepo

Italy

Barry Palmer

New Zealand

Matthew Panagiotou

Greece

Anil Pandit

United States of America

Constantinos Pantos

Greece

Guido Parodi

Italy

Jeetesh Patel

United Kingdom

Matthias Pauschinger

Germany

Marco Pavani

Italy

Charles Pearman

United Kingdom 
Dudley Pennell

United Kingdom

Rebecca Perry

Australia

Pawel Petkow Dimitrow
Poland

Elisa Picardi

Italy

Claudio Picariello

Italy

\section{Stefano Pidello}

Italy

Anna Pilbrow

New Zealand

Bertram Pitt

United States of America

Carmine Pizzi

Italy

Alberto Polimeni

Italy

Katrina Poppe

New Zealand

Janet Powell

United Kingdom

Jeppe Praetorius

Denmark

David Preiss

United Kingdom

Riccardo Proietti

Italy

Armando Pucciarelli

Italy

Rajesh Puranik

Australia

Rishi Puri

Canada

Giorgio Quadri

Italy

Alicia Quirós

Spain

Amresh Raina

United States of America

Sushant Ranadive

United States of America
Eleni Rapsomaniki

United Kingdom

Brian Rayner

South Africa

David Rehkopf

United States of America

Giuseppe Rengo

Italy

Paul Rheeder

South Africa

Angelos Rigopoulos

Germany

Guillermo Rodrigo

Spain

Isabel Rodriguez

Spain

Bruno Roseguini

Brazil

Francesco Rotondi

Italy

Santiago Roura

Spain

Jason Roy

United States of America

Erdal Safak

Germany

Samir Saha

Sweden

Tayfun Sahin

Turkey

Gianmario Sambuceti

Italy

Ana Santos

Portugal

Kátia Santos

Brazil

Nizal Sarrafzadegan

Iran

Filippo Sarullo

Italy

Hiroyuki Sasai

United States of America

Patrick Savage

United States of America
Ana Savic-Radojevic

Serbia

Hartzell Schaff

United States of America

Bruno Scheller

Germany

Francois Schellevis

Netherlands

Thomas Schindler

United States of America

Markus Schneider

Germany

Andreas Schoenenberger

Switzerland

Paul Schoof

Netherlands

Mikkel Malby Schoos

Denmark

Barbara Schumann

Sweden

Gregory Schwartz

United States of America

Robert Scragg

New Zealand

David Segal

Australia

Jitka Seidlerová

Czech Republic

Christine Seidman

United States of America

Gregory Sgueglia

Italy

Ryan Sheldon

United States of America

Motohiro Shimizu

Japan

Toshiharu Shinoka

United States of America

Supriya Shore

United States of America

Mohamed Shoukri

Saudi Arabia

Curt Sigmund

United States of America 
Isaac Silva

Brazil

\section{Mauro Silvestrini}

Italy

Andrew Sindone

Australia

Mukesh Singh

United States of America

\section{Asru Kumar Sinha}

India

Neil Smart

Australia

Wayne Smith

South Africa

Michelle Snyder

United States of America

Yoshimitsu Soga

Japan

Steinar Solberg

Norway

Hong Ji Song

Korea, South

Alexandra Sousa
Portugal
Adrian Specogna
Canada
Anthony Staines
Ireland
George Stavridis
Greece
Giulio Stefanini
Switzerland
Pierluigi Stefano
Italy
Paul Stein
United States of America
Louis-Mathieu Stevens
Canada
Welma Stonehouse
New Zealand
Stefan Stortecky
Switzerland
Richard Sutton
Monaco

Marijana Tadic

Serbia

Genzou Takemura

Japan

Yodo Tamaki

Japan

Weihong Tang

United States of America

Manabu Taniguchi

Japan

Bamidele O. Tayo

United States of America

Antonio Tello-Montoliu Spain

Martin Teraa

Netherlands

Sermin Tetik

Turkey

Randall Thompson

United States of America

Jakob Hartvig Thomsen

Denmark

Rebecca Thornhill

Canada

Jingwei Tian

China

Yaping Tian

China

Kimimasa Tobita

United States of America

Giuliano Tocci

Italy

Salvatore Tomasello

Italy

Per Tornvall

Sweden

Richard Toshner

United Kingdom

Lavinia Tran

Australia

Danijela Trifunovic

Serbia

Dimitrios Tsiapras

Greece
Karam Turk-Adawi

United States of America

Dimitrios Tziakas

Greece

Ioanna Tzoulaki

Greece

Anetta Undas

Poland

Roland Van Kimmenade

Netherlands

Johannes Van Rooyen

South Africa

Niels Van Royen

Netherlands

Moniek Van Zitteren

Netherlands

Praveen Veerabhadrappa

United States of America

Alessandro Verde

Italy

Richard Verrier

United States of America

Isabelle Vivodtzev

France

Veronica Ines Volberg

Argentina

Clemens Von Schacky

Germany

Franz Von Ziegler

Germany

Carina Wallgren-Pettersson

Finland

Haixia Wang

United States of America

Daowen Wang

China

Mingyi Wang

United States of America

Tzung-Dau Wang

Taiwan

Ralf Wassmuth

Germany

Lewis Watson

United States of America 
Heinz Weber

Austria

Bin Wei

United States of America

Lei Wei

United States of America

Niels Wiinberg

Denmark

Lars Wiklund

Sweden

Robert Wojciechowski

United States of America

Elizabeth Woodcock

Australia

\section{Zonggui Wu}

China

Ameeta Yaksh

Netherlands
Tomohide Yamada

Japan

Chih-Yu Yang

Taiwan

Yuichiro Yano

United States of America

Roseanne Yeung

Canada

Ali Yilmaz

Germany

Kai Hang Yiu

Hong Kong

Shi-Min Yuan

China

\section{Kyeong Ho Yun}

Korea, South

Katherine Yutzey

United States of America
Xinbo Zhang

United States of America

Luxia Zhang

China

Yuntao Zhao

China

Yinjun Zhao

United States of America

Stefan Zimmermann

Germany

Received: 14 January 2015 Accepted: 14 January 2015 Published: 7 April 2015

doi:10.1186/1471-2261-15-2

Cite this article as: Shipley: BMC Cardiovascular

Disorders reviewer acknowledgement 2014.

BMC Cardiovascular Disorders 2015 15:2. 